\title{
THE COHOMOLOGY OF TRANSITIVE FILTERED MODULES. I: THE FIRST COHOMOLOGY GROUP
}

\author{
BY \\ CHARLES FREIFELD $\left({ }^{1}\right)$
}

We are concerned here with the first cohomology groups of certain filtered modules over filtered Lie algebras. The basic generalities concerning these objects can be found in Rim [2].

Definition 1. A filtered Lie algebra is a Lie algebra $L$ together with a decreasing filtration $L^{-1} \supset L^{0} \supset L^{1} \supset \ldots \supset L^{n} \supset \ldots$ such that $\left[L^{i}, L^{j}\right] \subset L^{i+j}$ for all $i, j$.

The filtered Lie algebra $L$ is said to be transitive if

(a) $\bigcap_{i} L^{i}=\{0\}$,

(b) $L$ is complete in the topology given by the filtration, and

(c) $x \in L^{i}$ and $\left[L^{-1}, x\right] \subset L^{i}$ implies $x \in L^{i+1},(i \geqq 0)$.

Transitive filtered Lie algebras are treated more fully in [1], [2], [3] and [4]. We will assume always that dimension $L / L^{0}$ is finite.

Definition 2. A filtered module $M$ over a filtered Lie algebra $L$ is a triple $(L, M, \theta)$ where $\theta$ is a representation of $L$ on $M$ and $\theta\left(L^{i}\right) M^{j} \subset M^{i+j}$, for all $i, j$. Here $M$ is filtered $M^{-1} \supset M^{0} \supset M^{1} \supset \ldots \supset M^{n} \supset \ldots$. Thus, the representation is continuous with respect to the filtration topologies.

A transitive filtered $L$-module $M$ is a filtered $L$-module which has the following property: if $m \in M^{i}$ and $\theta(L) m \subset M^{i}$, then $m \in M^{i+1}(i \geqq 0)$.

In this paper, we will consider transitive $L$-modules $M$ with the additional hypothesis that $M$ be complete (with respect to the filtration topology) and separated (i.e., $\bigcap_{i} M^{i}=\{0\}$ ).

The Lie algebra cohomology groups for these spaces are defined as usual, except that one makes the requirement that all cochains be continuous with respect to these filtration topologies (see [2]). Here we will deal with $H^{1}(L, M)$, the first cohomology group, so that we will consider 1-cochains $c: L \rightarrow M$ which are continuous maps. A 1-cocycle $c$ satisfies (for all $x, y \in L$ )

$$
0=\partial c(x, y)=\theta(x) c(y)-\theta(y) c(x)-c([x, y]),
$$

and $c$ is a coboundary if there exists $d \in M$ such that

$$
\partial d(x)=\theta(x) d=c(x), \text { for all } x \in L .
$$

Received by the editors November 27, 1968 and, in revised form, February 12, 1969.

( $\left.{ }^{1}\right)$ This work represents most of the results obtained in the author's dissertation at Harvard University. The author wishes to thank Professors S. Sternberg and V. Guillemin for many helpful conversations. 
Furthermore, since both $L$ and $M$ are filtered, we can define the degree of a continuous linear mapping. We will say the degree $c \geqq k$ if $c\left(L^{j}\right) \subset M^{j+k}$ for all $j$ (see [2]). The coboundary operator is compatible with this and we denote by $H^{1}(L, M)_{q}$ the first cohomology group obtained when only cochains of degree at least $q$ are used. Finally if $(L, M, \theta)$ is an $L$-module, we denote by ( $\operatorname{gr} L, \operatorname{gr} M, \theta)$ the associated graded gr $L$-module [2].

Our primary concern is the case in which the transitive filtered Lie algebra $L$ is primitive, that is, $L^{0}$ is a maximal subalgebra [1], [5]. The methods used will rely on knowledge of the structure of these algebras, so that the results are valid for algebras with the same structure over all fields of characteristic zero. Over the complex numbers, it is known [5] that every infinite-dimensional primitive filtered Lie algebra is one of the following:

(1) $L_{C^{n}, \mathrm{Bl}(n, C)}$; the set of germs of all formal vectors fields on $C^{n}$,

(2) $L_{C^{n}, \text { sl }(n, C)}$; the set of germs of all volume-preserving formal vector fields on $C^{n}$, the volume element being $d x_{1} \wedge \cdots \wedge d x_{n}$,

(3) $L_{C^{n}, \mathrm{gl}(n, C), \mathrm{sl}(n, C)}{ }^{(1)}$; the set of germs of all formal power series vector fields on $C^{n}$ which preserve the volume element up to a constant factor,

(4) $L_{C^{2 n}{ }_{\text {,sp }}(n, C)}$; the set of germs of formal vector fields on $C^{2 n}$ which preserve a given closed exterior two-form of maximal rank (a symplectic structure), i.e., the infinitesimal canonical transformations,

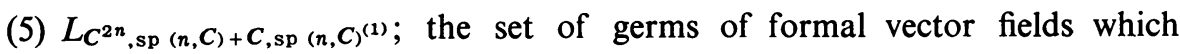
preserve the Hamiltonian form up to a constant factor, and

(6) the contact algebra; the set of germs of formal vector fields on $C^{2 n+1}$ which preserve the form $d z+\sum_{j=1}^{n}\left(y_{j} d x_{j}-x_{j} d y_{j}\right)$ up to a function factor.

In the following it will often be convenient to assume that $L$ has been realized as a subalgebra of the algebra of all formal power series as described in [1].

The primitive Lie algebras $L$ we are considering all have certain simple finitedimensional subalgebras $U$ of the isotropy algebra $L^{0}$ (except for $L_{C, \mathrm{Bl} \text { (1) }}$, which will be treated separately). In fact, if we filter $U$ as $U \supset U \supset\{0\}$, it will be a filtered subalgebra of $L$ if we take $U$ to be sl $(n, C)$ in cases (1), (2), (3) above and to be $\operatorname{sp}(n, C)$ in cases (4), (5), (6).

PROPOSITION 1. Let $U \supset U \supset\{0\}$ be a finite-dimensional filtered simple Lie algebra, and $M$ a filtered $U$-module with dimension $M / M^{0}$ finite. Then $H^{1}(U, M)=0$.

Proof. The conclusion is equivalent to showing $H^{1}(U, M)_{q}=0$ for all $q$, because $U$ has a finite filtration (in fact, every cocycle must be of degree at least -1 ). By the results of $\operatorname{Rim}$ [2], it therefore suffices to show $H^{1, q}(\operatorname{gr} U, \operatorname{gr} M)=0$ for all $q$, where $H^{1, q}$ means only 1-cocycles homogeneous of degree $q$ are used. But a homogeneous 1-cocycle of degree $q$ in this case is just a 1-cocycle in $H^{1}\left(U, M_{q}\right)$ (where $M_{q}=\operatorname{gr}_{q} M=M^{q} / M^{q+1}$ ), which is the usual Lie algebra cohomology with coefficients in a finite-dimensional vector space, since $U$ is contained in the isotropy algebra. Now $H^{1}\left(U, M_{q}\right)=0$ for all finite-dimensional modules $M_{q}$. 
Note that if $U \subset L^{0}$ is a subalgebra of the isotropy algebra, it acts on cochains by

$$
(\theta(x) \cdot c)(y)=\theta(x) c(y)-c([x, y])
$$

for $x \in U, y \in L$. This action commutes with the coboundary and is compatible with the degree of a cochain (see [4] for proofs). Hence there is an action of $U$ on $H^{1}(L, M)_{q}$.

THEOREM 1. Let $U \supset U \supset\{0\}$ be a simple filtered subalgebra of $L$. Then $U$ acts trivially on the cohomology classes in $H^{1}(L, M)$, i.e., the cohomology classes are invariant under $U$.

Proof. Let $c$ be a cocycle representing some cohomology class in $H^{1}(L, M)$. Then we can restrict $c$ to $U \supset U \supset\{0\}$, and we obtain a cohomology class in $H^{1}(U, M)$. By Proposition 1, this cocycle is a coboundary. That is, there exists $d \in M$ such that $c(x)=\theta(x) d$, for all $x \in U$. Now for $x \in U, y \in L$,

$$
\begin{aligned}
\theta(x)(c-\theta(\quad) d)(y)= & \theta(x) c(y)-\theta(x) \theta(y) d-c([x, y])+\theta([x, y]) d \\
= & \theta(y) c(x)+c([x, y])-\theta(y) \theta(x) d-\theta([x, y]) d \\
& -c([x, y])+\theta([x, y]) d \\
= & \theta(y)(c(x)-\theta(x) d)=0,
\end{aligned}
$$

since $c(x)=\theta(x) d$ for all $x \in U$.

I. Let $(L, M, \theta)$ be a transitive filtered module with $M / M^{0}$ finite-dimensional, and $L$ primitive. We wish to show that $H^{1}(L, M)$ is finite-dimensional.

First of all, $H^{1}(L, M)_{q}$ is finite-dimensional, as follows from Rim [2], and known results on the Spencer cohomology groups [1]. Now we claim $H^{1}(L, M)=$ $\bigcup_{q} H^{1}(L, M)_{q}$, i.e., every 1-cocycle has some finite degree. Consider $(\operatorname{Ker} c)$ $+c^{-1}\left(M^{0}\right) \subset L$. This closed subspace is of finite codimension in $L$ since a complement to it is injected into a complement to $M^{0}$ in $M$. Hence, by the definition of the filtration topology, there exists an integer $k$ such that $L^{k} \subset(\operatorname{Ker} c)+c^{-1}\left(M^{0}\right)$ (a closed subspace of finite codimension is open). Thus, $c\left(L^{k}\right) \subset M^{0}$. Now we claim that $c\left(L^{k+i}\right) \subset M^{i}$. Consider the cocycle equation

$$
\theta(x) c(y)-\theta(y) c(x)=c([x, y])
$$

for $x \in L^{-1}, y \in L^{k+1} . \theta(y) c(x) \in M^{0}$ (either $k>-1$ or $k=-1$, in which case $\left.c\left(L^{0}\right) \subset M^{0}\right)$. $[x, y] \in L^{k}$, so $c([x, y]) \in M^{0}$. Hence $\theta(x) c(y) \in M^{0}$, for all $x \in L^{-1}$. Therefore $c(y) \in M^{1}$ by transitivity. Continuing in this way we obtain $c\left(L^{k+i}\right) \subset M^{i}$.

Thus, to show $H^{\prime}(L, M)$ is finite-dimensional, it suffices to show that $H^{1}(L, M)_{k}$ $=H^{1}(L, M)_{k-1}$ for $k$ sufficiently negative $(k \ll 0)$. (This approach shows also that the result will remain true if $L$ has arbitrary transitive filtration.)

We use the following notation. $g^{k}$ will denote a complement to $L^{k+1}$ in $L^{k}$. (Specific knowledge of the structure of $g^{k}$ will be used.) $M_{j}$ will denote a complement to $M^{j+1}$ in $M^{j}$.

Let $c$ be a 1 -cocycle of degree $k$. We must show that $c$ is zero $(k \ll 0)$ because there are no 1-coboundaries of degree less than -1 . We claim that it is sufficient 
to show that $c$ restricted to (any) $g^{-k-1}$ is zero. For suppose this is so. Since degree $c=k, c\left(L^{-k}\right) \subset M^{0}$. Now if $x \in g^{-1}, y \in g^{-k}$, we have

$$
\theta(x) c(y)-\theta(y) c(x)=c([x, y]) .
$$

Since $c\left(L^{-k-1}\right) \subset M^{0},[x, y] \in L^{-k-1}$, and $\theta(y) c(x) \in M^{j}$ (where $j$ is large when $k \ll 0$ ), it follows that $\theta(x) c(y) \in M^{0}$ for all $x \in g^{-1}$. This contradicts the transitivity of the $L$-module $M$ unless $c(y)=0$. Thus $\left.c\right|_{g^{-k}}$ is 0 . Repetition of this argument for $y \in g^{-k+1}$, etc., shows that $\left.c\right|_{g^{-k+j}}$ is 0 for $j \geqq-1$. Thus $c$ must have degree greater than or equal to $k+1$, as is immediate from the definition of degree.

THEOREM 2. Let $(L, M, \theta)$ be a transitive filtered module, with $L$ primitive and dimension $M / M^{0}$ finite. Then $H^{1}(L, M)$ is finite-dimensional.

Proof. First of all, if $L$ is finite-dimensional, we are done because, by the above remarks, we must only show that there are no 1-cocycles of large negative degree. This is obviously the case if $L$ is finite-dimensional.

The rest of the proof breaks into several cases. Let $k \ll 0$.

(a) $L$ is one of the primitive infinite algebras of type (2) or (3) above:

In (2), we may choose $g^{0}=\mathrm{sl}(n, C) \subset L^{0}$ and in (3), $g^{0}=\mathrm{gl}(n, C) \supset \mathrm{sl}(n, C)$. $g^{-k}=\operatorname{sl}(n, C)^{(-k)}$ (in the notation of [1]), the set of homogeneous polynomial vector fields of order $-k+1$ which preserve volume, and the action of $\operatorname{sl}(n, C)$ on $g^{-k}$ under restriction of the adjoint action of $L$ on itself is just the $(-k+2)$ nd symmetric power of the fundamental representation of $\operatorname{sl}(n, C)$ on $C^{n}$ (see [1], [3], or [9]).

Now the definition of 1-cocycles gives

$$
\theta(x) c\left(x_{-k-1}\right)-\theta\left(x_{-k-1}\right) c(x)=c\left(\left[x, x_{-k-1}\right]\right)
$$

where $x \in \operatorname{sl}(n, C), x_{-k-1} \in g^{-k-1} . \theta\left(x_{-k-1}\right) c(x) \in M^{j}$ with $j \geqq 0$ if $-k$ is large enough, so we obtain that $\theta(x) c\left(x_{-k-1}\right)$ and $c\left(\left[x, x_{-1}\right]\right)$ have the same $M_{-1}$ component, i.e. $\left({ }^{* *}\right) \theta(x) c\left(x_{-k-1}\right)=c\left(\left[x, x_{-1}\right]\right)$ modulo $M^{0}$. It follows that if $x_{-k-1}$ is a weight vector for the (irreducible) representation of $\mathrm{sl}(n, C)$ on $g^{-k-1}, c\left(x_{-k-1}\right)$ is a weight vector (of the same weight) for the representation of $\mathrm{sl}(n, C)$ on the projection of $c\left(g^{-k-1}\right)$ in $M_{-1}$. But as $-k$ increases, the maximal weight of the representation of $\mathrm{sl}(n, C)$ on $g^{-k-1}$ increases. This is impossible unless $c\left(g^{-k-1}\right)$ has zero $M_{-1}$ component for $k \ll 0$ since dimension of $M_{-1}$ is finite. Thus $c\left(g^{-k-1}\right)$ $\subset M^{0}$. It now follows immediately from the definition of 1-cocycle and the transitivity of the $L$-module that degree $c \geqq k+1$.

(b) $L$ is of type (4) or (5).

In (4), choose $g^{0}=\mathrm{sp}(n, C)$; in (5), sp $(n, C) \subset g^{0}=\mathrm{sp}(n, C)+C \subset L^{0}$. Analogously to case (a), the restriction of the adjoint representation of $L$ on itself is the $(-k+2)$ nd symmetric power of the fundamental representation of $\mathrm{sp}(n, C)$ on

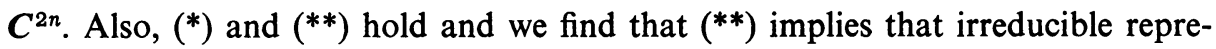
sentations of $\operatorname{sp}(n, C)$ of arbitrarily large dimension must be contained in $M_{-1}$ 
unless $c\left(g^{-k-1}\right)$ has zero $M_{-1}$ component. Therefore there are no cocycles of sufficiently high negative degree.

(c) $L$ is of type (1):

$L$ contains a transitive subalgebra of type (3), so we may assume that $c$ restricted to this subalgebra is of degree greater than $k$. We may choose $g^{0}=\mathrm{gl}(n, C)$. The finite-dimensional simple algebra $\mathrm{sl}(n, C)$ is contained in $g^{0}$, so its representation on $g^{-k-1}=\mathrm{gl}(n, C)^{(-k-1)}$ is completely reducible. Now we claim that $g^{-k-1}$ is the direct sum of two irreducible representations, namely sl $(n, C)^{-k-1}$ and $S^{-k-2}\left(C^{n}\right)$ (the symmetric power of $C^{n}$ ). This is seen as follows: let $x_{1}, \ldots, x_{n}$ denote coordinates in $\boldsymbol{C}^{n}$. We can represent a basis for the complement of $\mathrm{sl}(n, C)^{(-k-1)}$ in $g^{-k-1}$ by the monomial power series vector fields $x_{i_{1}}^{\alpha_{1}} \cdots x_{i_{s}}^{\alpha_{s}}\left(\partial / \partial x_{r}\right)$, where $\alpha_{1}+\cdots+\alpha_{s}=-k+j+1, \alpha_{i} \geqq 0,1 \leqq i \leqq s, 1 \leqq i_{t} \leqq n, 1 \leqq r \leqq n$, and it is assumed that $r=i_{1}$ if $x_{i_{1}}$ is the only variable appearing. Straightforward computation now implies that under the restriction of the adjoint action, $x_{i}\left(\partial / \partial x_{i}\right)$ acts with increasing weights on these elements. Therefore, by $\left({ }^{* *}\right)$, the representation of $g^{0}$ on the projection of $c\left(g^{-k-1}\right)$ on $M_{-1}$ has increasing weights and this implies that this projection must be zero since $M_{-1}$ is finite-dimensional.

(d) $L$ is of type (6):

We let $x_{1}, \ldots, x_{n}, y_{1}, \ldots, y_{n}, z$ be coordinates in $C^{2 n+1}$, and consider the subalgebra of $g^{0}$ consisting in the sum of $\operatorname{sp}(n, C)$ and

$$
Z_{0}=2 z \frac{\partial}{\partial z}+\sum_{i=1}^{n} x_{i} \frac{\partial}{\partial x_{i}}+\sum_{i=1}^{n} y_{i} \frac{\partial}{\partial y_{i}}
$$

We may choose ([3], [9])

$$
g^{-k-1}=Z_{-k-1} \oplus z^{-k-2} V_{1} \oplus z^{-k-3} \mathrm{sp}\left(V_{1}\right) \oplus \cdots \oplus \mathrm{sp}\left(V_{1}\right)^{(-k-1)},
$$

where $V_{1}$ is the vector space generated by $\left\{x_{1}, \ldots, x_{n}, y_{1}, \ldots, y_{n}\right\}$ and

$$
Z_{-k-1}=2 \frac{z^{-k}}{(-k) !} \frac{\partial}{\partial z}+\frac{z^{-k-1}}{(-k-1) !}\left(\sum_{j=1}^{n}\left(x_{j} \frac{\partial}{\partial x_{j}}+y_{j} \frac{\partial}{\partial y_{j}}\right)\right)
$$

Now the argument in case (b) shows that the projection of $c\left(\operatorname{sp}\left(V_{1}\right)^{(-k-1)}\right)$ on $M_{-1}$ must be zero. Direct computation shows that the action of $Z_{0}$ on the rest of $g^{-k-1}$ (under the restriction of the adjoint action) has increasing weights as $-k$ increases. This would imply the existence of infinitely many independent vectors in the finite-dimensional space $M_{-1}$, by (**). Q.E.D.

II. $L-F$ modules. Let $L$ be a transitive filtered Lie algebra. Denote by $F\left\{L / L^{0}\right\}$ the set of formal power series on the vector space $L / L^{0}$.

Definition 3. An $L-F$ module $E$ is a triple $(L, E, \theta)$ such that

(1) $\theta$ is a representation of the transitive filtered Lie algebra $L$ on a vector space $E$, 
(2) $E$ is an $F\left\{L / L^{0}\right\}$-module and

(3) $\theta(x)(f \cdot m)=x(f) \cdot m+f \cdot \theta(x) m$, where $x \in L, f \in F\left\{L / L^{0}\right\}, m \in E$.

In (3) it has been assumed that $L$ has been realized as a set of derivations of $F\left\{L / L^{0}\right\}$ (as can always be done ([1], [2])), and $x(f)$ means the action of $x \in L$ on the formal power series $F$. One defines $E^{i}=F^{i}\left\{L / L^{0}\right\} \cdot E$, where $F^{i}$ denotes the formal power series vanishing to order $i$. If $\bigcap_{i} E^{i}=\{0\}$, we say that the $L-F$ module $E$ is transitive. These modules should be thought of as the formal versions of the module of local sections of a vector bundle over a finite-dimensional manifold. In fact, condition (3) of the definition is just the infinitesimal form of the imprimitivity condition of Mackey [6]. In [7], it is proved that these modules are induced from representations of the isotropy algebra $L^{0}$, giving a formal version of Mackey's imprimitivity theorem. Finally, it should be noted that if $E$ is a finitely-generated $F\left\{L / L^{0}\right\}$-module, then Nakayama's lemma (or Krull's lemma) implies that $E$ is free with basis in a complement to $E^{0}$ and transitive ([7]).

TheOREM 2. Let $(L, E, \theta)$ be a transitive $L-F$ module and assume gr $E$ is generated by $\mathrm{gr}_{-1} E$ as an $F\left\{L / L^{0}\right\}$-module. Then $H^{1}(L, E)_{0}=0$.

Proof. It suffices to show that $H^{1, k}(\operatorname{gr} L, \operatorname{gr} E)=0$ for all $k \geqq 0$ : one has the exact sequence

$$
\cdots \rightarrow H^{1}(L, E)_{k+1} \rightarrow H^{1}(L, E)_{k} \rightarrow H^{1, k}(\operatorname{gr} L, \text { gr } E) \rightarrow \cdots
$$

which is the long exact cohomology sequence associated to the exact sequence

$$
0 \rightarrow \operatorname{Hom}(\wedge L, E)_{k+1} \rightarrow \operatorname{Hom}(\wedge L, E)_{k} \rightarrow \operatorname{Hom}^{k}(\wedge \operatorname{gr} L, \text { gr } E) \rightarrow 0
$$

where Hom $(\wedge L, E)_{k}$ denotes the linear maps from the exterior algebra $\wedge L$ to $E$ of degree at least $k$ [2] and $\mathrm{Hom}^{k}$ denotes maps homogeneous of degree $k$. Thus, if $H^{1, k}=0$, all $k \geqq 0$, we have $H^{1}(L, E)_{k+1} \rightarrow H^{1}(L, E)_{k}$ is surjective for all $k \geqq 0$. This is impossible, unless $H^{1}(L, E)_{0}=0$ since, as was noted before, every cocycle has a finite degree.

Let $\left\{e_{i}\right\}_{i \in I}$ be a set of generators for $\operatorname{gr}_{-1} E$. Let $\partial / \partial x_{1}, \ldots, \partial / \partial x_{n}$ denote a basis for $L / L^{0}$. We have $\theta\left(\partial / \partial x_{i}\right) e_{j}=0$ for $1 \leqq i \leqq n, j \in I$, in gr $E$, by definition of graded module. Now if $c$ is a cocycle of homogeneous degree greater than or equal to zero, it suffices, in order to show that it is a coboundary, to find $d \in \mathrm{gr} E$ such that $\theta\left(\partial / \partial x_{i}\right) d=c\left(\partial / \partial x_{i}\right), 1 \leqq i \leqq n$. For if this is so, then $c$ is a coboundary on $g^{-1}$ and the cocycle equation

$$
\theta\left(\frac{\partial}{\partial x_{i}}\right) c(y)-\theta(y) c\left(\frac{\partial}{\partial x_{i}}\right)=c\left(\left[\frac{\partial}{\partial x_{i}}, y\right]\right)
$$

for $y \in g^{0}=\mathrm{gr}_{0} L$ implies

$$
\theta\left(\frac{\partial}{\partial x_{i}}\right) c(y)-\theta(y) \theta\left(\frac{\partial}{\partial x_{i}}\right) d=c\left(\left[\frac{\partial}{\partial x_{i}}, y\right]\right),
$$


or

$$
\theta\left(\frac{\partial}{\partial x_{i}}\right)(c(y)-\theta(y) d)=c\left(\left[\frac{\partial}{\partial x_{i}}, y\right]\right)-\theta\left(\left[\frac{\partial}{\partial x_{i}}, y\right]\right) d
$$

Now since $\left[\partial / \partial x_{i}, y\right] \in g^{-1}$, we have

$$
\theta\left(\frac{\partial}{\partial x_{i}}\right)(c(y)-\theta(y) d)=0 \text { for all } 1 \leqq i \leqq n .
$$

This implies that $c(y)=\theta(y) d$ for all $y \in g^{0}$. Continuing this way, we obtain finally that $c(y)=\theta(y) d$ for all $y \in \operatorname{gr} L$. Thus it is sufficient to show $c$ is a coboundary when restricted to $g^{-1}$.

Let $c\left(\partial / \partial x_{i}\right)=\sum A_{i,}^{i} e_{i,}$ for $1 \leqq i \leqq n$, where $A_{i,}^{i}$ are formal power series in $n$ variables. If $d=\sum f_{s} e_{s} \in \mathrm{gr} E$ is a finite linear combination of basis elements with formal power series coefficients, then the definition of $L-F$ module gives

$$
\theta\left(\frac{\partial}{\partial x_{i}}\right) d=\sum_{s} \frac{\partial f_{s}}{\partial x_{i}} e_{s}+\sum_{s} f_{s} \theta\left(\frac{\partial}{\partial x_{i}}\right) \grave{e_{s}}=\sum_{s} \frac{\partial f_{s}}{\partial x_{i}} e_{s} .
$$

Thus, it suffices to solve the system $\partial f_{s} / \partial x_{i}=A_{s}^{i}$, for $1 \leqq i \leqq n$, and $s$ ranging through the possible $i_{j}$ occurring. It is obvious that we can solve this system because $f_{s}$ may be an arbitrary power series in $n$ variables; hence that $c$ is a coboundary.

RemarK. We may use Theorem 2 when gr $E$ is generated by $\left\{e_{i}\right\}$ in a topological sense, by observing that $f_{s}$ can be chosen to vanish to high order if $A_{s}^{i}$ does.

Definition 4. The dimension of an $L-F$ module $E$ is the dimension of $E / E^{0}$ as a vector space over the base field.

In general, $H^{1}(L, E)$ measures the rigidity of the representation, in terms of deformation theory [8]. When $E$ is one-dimensional (i.e., the formal sections of a line-bundle), there are two important cases. Let $\{e\}$, with $e \in E$, be a basis for $E$ over $F\left\{L / L^{0}\right\}$. We may assume that $e$ lies in a complement to $E^{0}$. Then we have

$$
\theta(x)(f e)=x(f) e+f \theta(x) e .
$$

Now $\theta(x) e=\rho(x) e$, where $\rho(x) \in F\left\{L / L^{0}\right\}$. An easy calculation shows that $\rho: L$ $\rightarrow F\left\{L / L^{0}\right\}$ is a 1-cocycle in $H^{1}\left(L, F\left\{L / L^{0}\right\}\right)$ and one can prove [7] that the cohomology classes in $H^{1}\left(L, F\left\{L / L^{0}\right\}\right)$ are in 1-1 correspondence with the inequivalent onedimensional $L-F$ modules. Here $L$ acts on $F\left\{L / L^{0}\right\}$ by being a set of derivations.

We want to calculate the above cohomology group for $L$ primitive.

THEOREM 3. Let $L$ be a primitive infinite-dimensional Lie algebra over the complex numbers and $F$ denote the standard $L-F$ module $F\left\{L / L^{0}\right\}$. Then

$$
\begin{aligned}
H^{1}(L, F) & =C \quad \text { if } L \text { is of type (1), (3), (5), or (6) } \\
& =0 \quad \text { if } L \text { is of type (2), (4). }
\end{aligned}
$$

Proof. The 1-cocycles of nonnegative degree are coboundaries by Theorem 2 . 
Let $L$ be of type (2) or (4). Let $c$ be a 1-cocycle of degree $-k, k>0$. The cocycle equation reads

$$
\theta(x) c(y)-\theta(y) c(x)=c([x, y])
$$

for $x \in \operatorname{sl}(n, C) \quad($ or $x \in \operatorname{sp}(n, C)) \subset L^{0}, y \in \operatorname{sl}(n, C)^{(k-1)} \quad\left(\right.$ or $\left.y \in \operatorname{sp}(n, C)^{(k-1)}\right)$ $\subset L^{k-1}$. The action of $\mathrm{sl}(n, C)$ (or $\mathrm{sp}(n, C)$ ) on the one-dimensional subspace of constants must be trivial because $\mathrm{sl}(n, C)$ (or $\mathrm{sp}(n, C))$ is a simple algebra. Consider first the case in which $k \geqq 2$. Then $\theta(y) c(x) \in F^{0}$ because $c(x) \in F$ and $y \in L^{1}$. Therefore, on the subspace of constants we have (for $\left.x \in \operatorname{sl}(n, C), y \in \operatorname{sl}(n, C)^{(k-1)}\right)$

$$
\theta(x) c(y)=c([x, y]) .
$$

But we know that under the restriction of the adjoint action, $\mathrm{sl}(n, C)$ (or sp $(n, C)$ ) acts irreducibly and with nontrivial maximal weight on $\operatorname{sl}(n, C)^{(k-1)}$ (or sp $\left.(n, C)^{(k-1)}\right)$. Thus the action of $\operatorname{sl}(n, C)$ (or sp $\left.(n, C)\right)$ cannot be trivial on the constants unless $c\left(\operatorname{sl}(n, C)^{(k-1)}\right)=\{0\}\left(c\left(\operatorname{sp}(n, C)^{(k-1)}\right)=\{0\}\right) \bmod F^{0}$. As before, $\left(^{*}\right)$ implies that degree $c \geqq-k+1$.

Now let $c$ have degree -1 . Since $\operatorname{sl}(n, C)(\operatorname{sp}(n, C))$ is simple and contained in $L^{0}, c$ is a coboundary when restricted to $\operatorname{sl}(n, C)(\operatorname{sp}(n, C))$. We can therefore subtract a coboundary term from $c$ to obtain a cocycle $\bar{c}$ of degree -1 which vanishes on $\mathrm{sl}(n, C)(\operatorname{sp}(n, C))$. This implies immediately that $\bar{c}$ must be of degree $\geqq 0$.

(b) $L$ is of type (1), (3), (5), or (6).

First let $L$ be of type (1). If dimension $L / L^{0}>1$, we have sl $(n, C) \subset L^{0}$ and the restriction of the adjoint representation of $\operatorname{sl}(n, C)$ on $\mathrm{gl}(n, C)^{(k)}$ is completely reducible. If $k \geqq 2$, the cocycle equation $\left(^{*}\right)$ implies that $c\left(\mathrm{gl}(n, C)^{(k)}\right)=\{0\} \bmod F^{0}$ as before. Hence there are no cocycles of degree $\leqq-2$. If degree $c=-1$, observe that we can assume $c$ is a coboundary on the transitive subalgebra of $L$ of type (2). That is, we may assume $c$ vanishes on $\operatorname{sl}(n, C) \subset \mathrm{gl}(n, C) \subset L^{0}$. This leaves at most a one-dimensional space of nontrivial cocycles since a cocycle of degree -1 is determined by its restriction to $\mathrm{gl}(n, C)$.

If $n=1$ above, the equation $\left(^{*}\right)$ implies directly that there are no cocycles of degree $\leqq-2$. Since $\mathrm{gl}(1, \boldsymbol{C})$ is one-dimensional, there is at most a one-dimensional set of nontrivial cocycles (of degree $=-1$ ).

If $L$ is of type (3) or (5), it contains a transitive subalgebra of type (2) or (4) and of codimension one. This implies that there is at most a one-dimensional space of nontrivial cocycles.

Let $L$ be of type (6). We will denote the vector fields in the realization of the algebra $L$ with the variables $x_{1}, \ldots, x_{n}, y_{1}, \ldots, y_{n}, z$, where $V_{1}=\left\{x_{1}, \ldots, x_{n}\right.$, $\left.y_{1}, \ldots, y_{n}\right\}$ is the subspace of codimension one defined by the contact form. Let $c$ be a cocycle of negative degree. We can always assume that $c$ vanishes on $\operatorname{sp}(n, C)$ which is contained in the linear isotropy algebra. We view the linear isotropy algebra as contained in $L$, using the results in Sternberg [9]. The linear isotropy 
algebra $g^{0}$ is a semidirect product of the simple algebra $\mathrm{sp}(n, C)$ and the radical of $g^{0}$, which is a $(2 n+1)$-dimensional vector space spanned by the vector fields $(i=1, \ldots, n)$

$$
\begin{aligned}
& z \frac{\partial}{\partial x_{i}}+y_{i}\left(\sum_{j=1}^{n} x_{j} \frac{\partial}{\partial x_{j}}+\sum_{j=1}^{n} y_{j} \frac{\partial}{\partial y_{j}}+z \frac{\partial}{\partial z}\right), \\
& z \frac{\partial}{\partial y_{i}}-x_{i}\left(\sum_{i=1}^{n} x_{j} \frac{\partial}{\partial x_{j}}+\sum_{i=1}^{n} y_{j} \frac{\partial}{\partial y_{j}}+z \frac{\partial}{\partial z}\right) \\
& 2 z \frac{\partial}{\partial z}+\sum_{j=1}^{n} x_{j} \frac{\partial}{\partial x_{j}}+\sum_{i=1}^{n} y_{j} \frac{\partial}{\partial y_{j}} .
\end{aligned}
$$

We consider first the case in which $c$ has degree less than $-1 . L^{0}$ contains $\operatorname{sp}(n, C)+\operatorname{sp}(n)^{(1)}+\cdots+\operatorname{sp}(n)^{(k)}+\cdots$, and as in the case of the algebra $L_{C^{2 n}, \operatorname{sp}(n, C)}$, the cocycle must carry sp $(n)^{(k-1)}$ into $F^{0}$, if $c$ is of degree $-k$ : the representation of $\operatorname{sp}(n, C)$ on sp $(n, C)^{(k-1)}$ has at least two nontrivial weight vectors and the lowest degree part of $F$ is one-dimensional. We denote this part by $F_{-1}$, which is a complement to $F^{0}$ in $F$. Now we make use of the explicit form of a complement to $L^{k}$ in $L^{k-1}$, as described in Sternberg [9], namely:

where

$$
g^{k-1}=\left\{Z_{k-1}\right\}+z^{k} V_{1}+z^{k-1} \mathrm{sp}\left(V_{1}\right)+\cdots+\mathrm{sp}\left(V_{1}\right)^{k-1},
$$

$$
Z_{k-1}=2 \frac{z^{k}}{k !} \frac{\partial}{\partial z}+\frac{z^{k-1}}{(k-1) !}\left(\sum x_{j} \frac{\partial}{\partial x_{j}}+\sum y_{j} \frac{\partial}{\partial y_{j}}\right),
$$

and $V_{1}$ is as before. Here $k \geqq 2$, since the degree $c$ is $\leqq-2$. Now we can use the representation of $\operatorname{sp}\left(V_{1}\right)$ on each of the factors of this direct sum which contains $V_{1}$ to conclude that $c$ must carry them into $F^{0}: \operatorname{sp}\left(V_{1}\right)$ acts irreducibly under the adjoint representation and has nontrivial weight vectors $(n>0)$ and the lowest order part of the image of $c$ is in a one-dimensional space. It remains to consider $c\left(Z_{k-1}\right)$. To do this, observe that $c$ carries the following vector fields into $F^{0}$, again because $\mathrm{sp}(n)$ acts irreducibly and with nontrivial weight vectors:

$$
\begin{aligned}
& A_{i}=z \frac{\partial}{\partial x_{i}}+y_{i}\left(\sum_{j=1}^{n} x_{j} \frac{\partial}{\partial x_{j}}+\sum_{j=1}^{n} y_{j} \frac{\partial}{\partial y_{j}}+z \frac{\partial}{\partial z}\right), \\
& B_{i}=z \frac{\partial}{\partial y_{i}}-x_{i}\left(\sum x_{j} \frac{\partial}{\partial x_{j}}+\sum y_{j} \frac{\partial}{\partial y_{j}}+z \frac{\partial}{\partial z}\right),
\end{aligned}
$$

$(i=1, \ldots, n)$. Now consider the bracket of $Z_{k-1}$ with these vectors, and the bracket of $Z_{0}$ with these vectors. From $\left[Z_{0}, A_{i}\right]=-A_{i}$, one obtains that if $c\left(Z_{0}\right)$ has a nonzero component in $\dot{F}_{-1}$, one must have $\theta\left(A_{i}\right) e=-e$ where $e$ is the generator of $F$. But then since $\left[Z_{k-1}, A_{i}\right]=r A_{i}$, where $r$ is a constant different from minus one, we have from

$$
\theta\left(A_{i}\right) c\left(Z_{k-1}\right)-\theta\left(Z_{k-1}\right) c\left(A_{i}\right)=r c\left(A_{i}\right)
$$

that the lowest order term of $c\left(Z_{k-1}\right)$ must be in $F^{0}$ (we would otherwise have 
$\theta\left(A_{i}\right) e=r e$, since $k>1$ here). There remains only the possibility that $c\left(Z_{0}\right) \subset F^{0}$. But then using $\left[Z_{0}, Z_{k-1}\right]=2(k-1) Z_{k-1}$, we obtain that only one of the $c\left(Z_{m}\right)$ may have a nonzero component in $F_{-1}$ (since, again, $F / F^{0}$ is one-dimensional), and that must be $c\left(Z_{k-1}\right)$ if $c$ is to be of degree $-k$. But we have

$$
\theta\left(\frac{\partial}{\partial z}\right) c\left(Z_{k-1}\right)=\theta\left(Z_{k-1}\right) c\left(\frac{\partial}{\partial z}\right)+c\left(Z_{k-2}\right)
$$

which implies that $\theta(\partial / \partial z) c\left(Z_{k-1}\right)$ has no nonzero component in $F_{-1}$, since nothing on the right side does, and

$$
\begin{aligned}
& \theta\left(z \frac{\partial}{\partial z}+\sum x_{j} \frac{\partial}{\partial x_{j}}+\sum y_{j} \frac{\partial}{\partial y_{j}}\right) c\left(Z_{k-1}\right) \\
& =\theta\left(Z_{k-1}\right) c\left(z \frac{\partial}{\partial z}+\sum x_{j} \frac{\partial}{\partial x_{j}}+\sum y_{j} \frac{\partial}{\partial y_{j}}\right)+2(k-1) c\left(Z_{k-1}\right)
\end{aligned}
$$

which shows that

$$
\theta\left(z \frac{\partial}{\partial z}+\sum x_{j} \frac{\partial}{\partial x_{j}}+\sum y_{j} \frac{\partial}{\partial y_{j}}\right)
$$

acts nontrivially on the image of $c\left(Z_{k-1}\right)$ in $F_{-1}$, and finally,

$$
\begin{aligned}
\theta\left(\frac{z^{2}}{2 !} \frac{\partial}{\partial z}+\frac{z}{1}\left(\sum x_{j}\right.\right. & \left.\left.\frac{\partial}{\partial x_{j}}+\sum y_{j} \frac{\partial}{\partial y_{j}}\right)\right) c\left(Z_{k-1}\right) \\
& =\theta\left(Z_{k-1}\right) c\left(\frac{z^{2}}{2 !} \frac{\partial}{\partial z}+z\left(\sum x_{j} \frac{\partial}{\partial x_{j}}+\sum y_{j} \frac{\partial}{\partial y_{j}}\right)\right)+\operatorname{sc}\left(Z_{k}\right)
\end{aligned}
$$

where $s$ is a nonzero constant, which shows that

$$
\theta\left(\frac{z^{2}}{2 !} \frac{\partial}{\partial z}+z\left(\sum x_{j} \frac{\partial}{\partial x_{j}}+\sum y_{j} \frac{\partial}{\partial y_{j}}\right)\right)
$$

acts trivially on the $F_{-1}$ component of $c\left(Z_{k-1}\right)$. But

which implies that

$$
\begin{aligned}
\theta\left(z \frac{\partial}{\partial z}+\sum x_{j}+\sum y_{j} \frac{\partial}{\partial y_{j}}\right) c\left(Z_{k-1}\right) & \\
= & \theta\left(\frac{\partial}{\partial z}\right) \theta\left(\frac{z^{2}}{2 !} \frac{\partial}{\partial z}+z\left(\sum x_{j} \frac{\partial}{\partial x_{y}}+\sum y_{j} \frac{\partial}{\partial y_{j}}\right)\right) c\left(Z_{k-1}\right) \\
& -\theta\left(\frac{z^{2}}{2 !} \frac{\partial}{\partial z}+z\left(\sum x_{j} \frac{\partial}{\partial x_{j}}+\sum y_{j} \frac{\partial}{\partial y_{j}}\right)\right) \theta\left(\frac{\partial}{\partial z}\right) c\left(Z_{k-1}\right)
\end{aligned}
$$

$$
\theta\left(z \frac{\partial}{\partial z}+\sum x_{j} \frac{\partial}{\partial x_{j}}+\sum y_{j} \frac{\partial}{\partial y_{j}}\right)
$$

acts trivially on the $F_{-1}$ component of $c\left(Z_{k-1}\right)$, which is a contradiction.

If $c$ is of degree -1 , we must look at $c$ restricted to the linear isotropy algebra. The representation of $\operatorname{sp}(n, C)$ on the lowest order terms of $c\left(g^{0}\right)$ is a representation 
of $\operatorname{sp}(n, C)$ on a one-dimensional space; hence it is the trivial representation. But the representation of $\operatorname{sp}(n, C)$ on the vector fields of the form

$$
\begin{aligned}
& z \frac{\partial}{\partial x_{i}}+y_{i}\left(\sum x_{j} \frac{\partial}{\partial x_{j}}+\sum y_{j} \frac{\partial}{\partial y_{j}}+z \frac{\partial}{\partial z}\right) \\
& z \frac{\partial}{\partial y_{i}}-x_{i}\left(\sum x_{j} \frac{\partial}{\partial x_{j}}+\sum y_{j} \frac{\partial}{\partial y_{j}}+z \frac{\partial}{\partial z}\right)
\end{aligned}
$$

is, by direct computation, the irreducible representation of $\operatorname{sp}(n, C)$ on $C^{2 n}$ given by viewing $\operatorname{sp}(n, C)$ as matrices (i.e., the fundamental representation). This representation, of course, has nontrivial weight vectors. Thus, since $\theta(x) c(y)$ $=c([x, y])$ for $x \in \operatorname{sp}(n, C), y \in g^{0}, c$ must be zero on this subspace of $g^{0}$. This leaves only the one-dimensional possibility of cocycles of degree -1 which do not vanish on the vector field

$$
2 z \frac{\partial}{\partial z}+\sum x_{j} \frac{\partial}{\partial x_{j}}+\sum y_{j} \frac{\partial}{\partial y_{j}} .
$$

The following cocycles are nontrivial, showing that $H^{1}(L, F)=C$ :

Type (1). $c\left(\sum_{i=1}^{n} f_{i}\left(\partial / \partial x_{i}\right)\right)=\sum_{i=1}^{n}\left(\partial f_{i} / \partial x_{i}\right) . c$ is not a coboundary because it vanishes on all vector fields with $f_{i}$ constant, so that if $d \in F$ and

$$
c(\quad)=\theta(\quad) d
$$

$d$ must be in a complement to $F^{0}$. But $\theta(\quad) d=0$. (Geometrically, if $c$ were a coboundary, there would exist a volume-form preserved by all infinitesimal motions, after a change in coordinates.)

Type (3). Same as (1).

Type (5). Define

Type (6). Define

$$
c\left(\sum_{i=1}^{n} f_{i} \frac{\partial}{\partial x_{i}}+\sum_{i=1}^{n} g_{i} \frac{\partial}{\partial y_{i}}\right)=\sum_{i=1}^{n}\left(\frac{\partial f_{i}}{\partial x_{i}}+\frac{\partial g_{i}}{\partial y_{i}}\right) .
$$

$$
c\left(\sum_{i=1}^{n} f_{i} \frac{\partial}{\partial x_{i}}+\sum_{i=1}^{n} g_{i} \frac{\partial}{\partial y_{i}}+h \frac{\partial}{\partial z}\right)=\sum_{i=1}^{n}\left(\frac{\partial f_{i}}{\partial x_{i}}+\frac{\partial g_{i}}{\partial y_{i}}\right)+\frac{\partial h}{\partial z}
$$

The methods of proof in Theorem 3 can be used to show that if $L$ is of type (2) or (4) and $E$ is an $L-F$ module of dimension less then dimension $g^{1}\left(=\operatorname{sl}(n, C)^{(1)}\right.$ or $\left.\mathrm{sp}(n, C)^{(1)}\right)$, then $H^{1}(L, E)=0$.

The situation is different when the one-dimensional bundle $E$ is not trivial. The following "vanishing" theorem can be proved.

THeOREM 4. Let $E$ be a one-dimensional $L-F$ module and $L$ a primitive infinitedimensional Lie algebra. Assume $E$ is not isomorphic to the standard $L-F$ module $F$. Then $H^{1}(L, E)=0$.

This theorem can be proved by direct computation as was Theorem 3 . It is found that the cohomology group is determined by its restriction to $g^{0}$, a complement to $L^{1}$ in $L^{0}$. (See [10] for details.) 
Much stronger results (than Theorems 3 and 4) on the cohomology of $L-F$ modules can be obtained using a consequence of the Frobenius Reciprocity Theorem. These will be presented in a forthcoming paper on the higher cohomology groups of transitive $L$-modules. (See Appendix of [11] for the Frobenius Reciprocity Theorem.)

III. The adjoint representation. Consider $(L, L, \theta)$ where $\theta$ is the adjoint representation. Then it follows easily from the definitions that $H^{1}(L, L)$ gives the space of continuous derivations which are not inner. What is this space when $L$ is a primitive infinite dimensional Lie algebra? First of all, observe that every such $L$ of type (2) or of type (4) is an ideal of codimension one in an $L$ of type (3) or (5). This implies immediately that for $L$ of type (2) or (4), dimension $H^{1}(L, L) \geqq 1$.

THEOREM 5. Let L be a primitive infinite-dimensional Lie algebra over the complex numbers and let $\theta$ be the adjoint representation. Then

$$
\begin{aligned}
H^{1}(L, L) & =0 \quad \text { if } L \text { is of type (1), (3), (5), (6), } \\
& =C \quad \text { if } L \text { is of type (2), (4). }
\end{aligned}
$$

Proof. The adjoint representation is a subrepresentation of an $L-F$ module. To see this, it suffices to show it for $L=L_{C^{n},{ }_{11(n, C)}}($ type (1)) because every $L$ is a transitive subalgebra of this one. Let $\left\{\partial / \partial x_{1}, \ldots, \partial / \partial x_{n}\right\}$ denote the basis of $L_{C^{n}, \mathbf{g l}(n, C)}$ as an $F\left\{\boldsymbol{C}^{n}\right\}$-module. Then define

$$
\theta\left(f \frac{\partial}{\partial x_{i}}\right)\left(\frac{\partial}{\partial x_{j}}\right)=-\frac{\partial f}{\partial x_{j}} \frac{\partial}{\partial x_{i}}=\left[f \frac{\partial}{\partial x_{i}}, \frac{\partial}{\partial x_{j}}\right]
$$

This defines the action of $L_{C^{n}, \mathrm{gl}(n, C)}$ on the basis of the $L-F$ module, and now it is easy to check that

$$
\begin{aligned}
\theta\left(\sum_{i=1}^{n} f_{i} \frac{\partial}{\partial x_{i}}\right)\left(\sum_{i=1}^{n} g_{i} \frac{\partial}{\partial x_{i}}\right) & =\sum_{i, j}\left(f_{i} \frac{\partial g_{i}}{\partial x_{i}} \frac{\partial}{\partial x_{j}}\right)-\sum_{i, j} g_{i} \theta\left(f_{j} \frac{\partial}{\partial x_{j}}\right) \frac{\partial}{\partial x_{j}} \\
& =\sum_{i, j} f_{i} \frac{\partial g_{j}}{\partial x_{i}} \frac{\partial}{\partial x_{j}}-\sum_{i, j} \frac{\partial f_{j}}{\partial x_{i}} \frac{\partial}{\partial x_{j}} \\
& =\sum_{j}\left(\sum_{i} f_{i} \frac{\partial g_{j}}{\partial x_{i}}-g_{i} \frac{\partial f_{j}}{\partial x_{i}}\right) \frac{\partial}{\partial x_{j}}
\end{aligned}
$$

which is indeed the bracket of the two vector fields. Thus, those arguments on the cohomology of $L-F$ modules in which we can show that certain cocycles must be identically zero (rather than being coboundaries) can be used without change in the present situation. For example, we can conclude that $H^{1}(L, L)_{k}=H^{1}(L, L)_{k-1}$ for $k \leqq-2$, (that is, there are no nontrivial cocycles of degree $\leqq-2$ ), as follows: Let $c: L \rightarrow L$ be a 1 -cocycle of degree $k \leqq-2$. Consider the cocycle equation

$$
[x, c(y)]-[y, c(x)]=c([x, y])
$$

for $x$ in (the subalgebra of $L$ isomorphic to) the linear isotropy algebra and $y \in L^{j}$, 
where $j \geqq 1$. Then $[y, c(x)] \in L^{0}$ so that on a complement $L_{-1}$ to $L^{0}$ in $L^{-1},\left(^{*}\right)$ becomes

$$
[x, c(y)]=c([x, y]) .
$$

But $\theta$ is the adjoint representation. That is, we know precisely what the representation of the linear isotropy algebra on $L_{-1}$ must be in each case. However, $\left({ }^{* *}\right)$ implies that the representation of the linear isotropy algebra on the image of $c$ in $L_{-1}$ must agree with the representation of the linear isotropy algebra on $L^{j} / L^{j+1}$ for some $j \geqq 1$. Case by case checking, using the information on the higher order structure of the $L$ 's given in the proof of the Theorems 2 and 3, shows immediately that no subrepresentation of this representation is contained in the representation of the linear isotropy algebra on $L_{-1}$. Hence, $c$ restricted to a complement to $L^{-k}$ in $L^{-k-1}$ must be zero. By transitivity, one concludes that degree $c>k$.

The remainder of the proof breaks into cases:

(a) $L$ is of type (2). Let $c$ be a 1-cocycle of degree $\geqq-1$. By Proposition $1, c$ is a coboundary on $g^{0}=\mathrm{sl}(n, C) \subset L^{0}$. By subtracting, we can take $c$ to be zero on $g^{0}$. Now it follows from the definition of transitivity that $c$ cannot have degree -1 . Let $c$ have nonnegative degree. Such a cocycle is determined when it is known on $g^{-1}$, a complement to $L^{0}$ in $L$. Since $c$ is zero on $\mathrm{sl}(n, C)$, the cocycle equation becomes

$$
[x, c(y)]=c([x, y])
$$

for $x \in \operatorname{sl}(n, C) \subset L^{0}$ and $y \in g^{-1}$. But the representation of $\mathrm{sl}(n, C)$ on $g^{k}$, a complement to $L^{k+1}$ in $L^{k}$, is known for the adjoint representation. It is just the $(k+1)$ st symmetric power of the fundamental representation of $\operatorname{sl}(n, C)$ on $\boldsymbol{C}^{n}$. The cocycle equation $\left(^{*}\right)$ then implies, if the degree of $c$ is zero, that the components of the elements $c\left(g^{-1}\right)$ in $g^{-1}$ must be completely determined. That is, there can be at most one nontrivial cocycle of degree exactly zero. We have given it above.

Now if degree $c=k>0$, we note that $\left(^{*}\right)$ implies that $(k+1)$ st symmetric power of the fundamental representation must contain the fundamental representation as a subrepresentation (namely, on the elements $c(y)$ for $y \in g^{-1}$ ). This is impossible, since the $(k+1)$ st symmetric power is irreducible.

(b) $L$ of type (4). Proceed precisely in the same manner as part (a), replacing $g^{0}=\operatorname{sl}(n, C)$ by $g^{0}=\operatorname{sp}(n, C)$.

(c) $L$ of type (3) or (5). Use the same argument as in parts (a) and (b). Note that the equation $\left(^{*}\right)$ eliminates the case degree $c>0$ in type (3), since the adjoint representation of $\mathrm{gl}(n, C)$ on itself does not contain the fundamental representation of $\mathrm{sl}(n, C)$. A direct argument handles the case degree $c=0$ and since $c$ vanishes on sl $(n, C)$, there is at most a one-dimensional possibility for nontrivial cocycles of degree -1 . But $(*)$ implies that $\operatorname{sl}(n, C)$ must act trivially on $c\left(\sum x_{i}\left(\partial / \partial x_{i}\right)\right)$ because $\operatorname{sl}(n, C)$ acts trivially on $\sum x_{i}\left(\partial / \partial x_{i}\right)$. Thus $c\left(\sum x_{i}\left(\partial / \partial x_{i}\right)\right)=0$.

Type (4) is handled similarly. 
(d) $L$ of type (1). Theorem 2 implies that $H^{1}(L, L)_{0}=0$ since $L$ is an $L-F$ module. Or we may argue as follows: Let degree $c \geqq 0$. $L$ is a flat algebra, i.e., $L$ is isomorphic to (the completion of) its associated graded algebra. Furthermore, for this $L$, it is known that the Spencer cohomology groups $H^{1, k}\left(\mathrm{gr}_{-1} L\right.$, gr $\left.L\right)$ vanish $(k \geqq 0)$ [1]. The work of [2] now implies immediately that $H^{1}(L, L)_{0}=0$, i.e., that there are no nontrivial cocycles of nonnegative degree.

Let degree $c=-1$. If dimension $L / L^{0}>1$, use the argument above in part (c) for cocycles of degree -1 . If $\operatorname{dim} L / L^{0}=1$, proceed as follows: After multiplying by a constant, we can assume that

$$
c(x d / d x)=d / d x
$$

for if $c(x d / d x)$ had a trivial projection in $g^{-1}$, the definition of cocycles would imply that degree $c \geqq 0$. But

so that the cocycle

$$
d / d x=[x d / d x,-d / d x]
$$

$$
c(\quad)-[,-d / d x]
$$

vanishes on $g^{0}$ and hence is a cocycle of degree $\geqq 0$. Therefore it is a coboundary, which of course implies that $c$ is a coboundary.

(e) $L$ of type (6). By [1], we can conclude that $H^{1}(L, L)_{k}=0$ for $k>0$. We assume as usual that $\left.c\right|_{\mathrm{sp}(n, C)}=0$. Then since the action of $\mathrm{sp}(n)$ on the elements in $c\left(g^{-1}\right)$ is the restriction of the adjoint action of $\operatorname{sp}(n)$ on $g^{-1}$, we conclude that

$$
\begin{aligned}
c\left(\frac{\partial}{\partial x_{i}}+y_{i} \frac{\partial}{\partial z}\right) & =r_{i}\left(\frac{\partial}{\partial x_{i}}+y_{i} \frac{\partial}{\partial z}\right), \\
c\left(\frac{\partial}{\partial y_{i}}-x_{i} \frac{\partial}{\partial z}\right) & =s_{i}\left(\frac{\partial}{\partial y_{i}}-x_{i} \frac{\partial}{\partial z}\right), \\
c\left(\frac{\partial}{\partial z}\right) & =t \frac{\partial}{\partial z}
\end{aligned}
$$

$i=1, \ldots, n$, where $r_{i}, s_{i}, t$ are constants, and where $g^{-1}$ now denotes the complement to $L^{0}$ in $L$ generated by

$$
\frac{\partial}{\partial x_{i}}+y_{i} \frac{\partial}{\partial z}, \quad \frac{\partial}{\partial y_{i}}-x_{i} \frac{\partial}{\partial z}, \quad \frac{\partial}{\partial z}
$$

$i=1, \ldots, n$, the equality holding after projection on $g^{-1}$. Now we have

$$
\left[\frac{\partial}{\partial x_{i}}+y_{i} \frac{\partial}{\partial z}, s_{i}\left(\frac{\partial}{\partial y_{i}}-x_{i} \frac{\partial}{\partial z}\right)\right]-\left[\frac{\partial}{\partial y_{i}}-x_{i} \frac{\partial}{\partial z}, r_{i}\left(\frac{\partial}{\partial x_{i}}+y_{i} \frac{\partial}{\partial z}\right)\right]=-2 t \frac{\partial}{\partial z}
$$

from the cocycle equation, (where we have used knowledge of $L^{0}$ to conclude that no new coefficients of $\partial / \partial z$ can enter), which implies that $s_{i}+r_{i}=t$. Also since

$$
\left[x_{i} \frac{\partial}{\partial y_{i}}, c\left(\frac{\partial}{\partial x_{i}}+y_{i} \frac{\partial}{\partial z}\right)\right]=c\left(\left[x_{i} \frac{\partial}{\partial y_{i}}, \frac{\partial}{\partial x_{i}}+y_{i} \frac{\partial}{\partial z}\right]\right)
$$


we have $s_{i}=r_{i}$, because

$$
\left[x_{i} \frac{\partial}{\partial y_{i}}, \frac{\partial}{\partial x_{i}}+y_{i} \frac{\partial}{\partial z}\right]=-\frac{\partial}{\partial y_{i}}+x_{i} \frac{\partial}{\partial z} .
$$

Thus, $s_{i}=r_{i}=t / 2$ and $c$ agrees with the coboundary $[, d]$ on $g^{-1}$,

$$
d=\frac{t}{2}\left(2 z \frac{\partial}{\partial z}+\sum\left(x_{j} \frac{\partial}{\partial x_{j}}+y_{j} \frac{\partial}{\partial y_{j}}\right)\right) \text {. }
$$

Hence $c()-[, d]$ is a cohomologous cocycle of degree $>0$, and is therefore a coboundary.

Method II. This uses the same arguments on the cocycles of degree -1 , and an observation of V. Guillemin for the cocycles of degree $\leqq-2$ or $\geqq 0$. The observation is that the contact algebra may be obtained as follows. Consider a manifold of dimension $2 n+1, M$, with a one-form $\omega$ of maximal rank (defined locally on $M$ ). Let $l$ be the line bundle over $M$ generated by $\omega$. It can be shown that the set of germs of local sections (at a point of $M$ ) of $l^{*}$ is a simple Lie algebra, where $l^{*}$ is the dual bundle and the bracket is given as follows: if $f, g$ are germs of sections of $l^{*}$ they can be viewed as germs of functions on $l$ which are linear in each fiber; the manifold $l$ is $(2 n+2)$-dimensional and the bracket is simply the Poisson bracket. It is not difficult to show that this algebra must be the contact algebra-one eliminates the other possibilities. The contact algebra then can be obtained as vector fields by looking at the symplectic manifold $l$ with Hamiltonian 2-form $\Omega=d \tilde{\omega}$, (where $\left.\pi: l \rightarrow M, \tilde{\omega}=\pi^{*} \omega\right)$ and using $\Omega$ to produce vector fields on $l$ corresponding to the functions on $l$. Since the contact algebra $l$ is the set of all sections of $l^{*}$, it is a module (one-dimensional) over the functions on $M$. It can now be shown that the adjoint action makes $L$ into an $L-F$ module. Now we can obtain $H^{1}(L, L)_{0}=0$ because we know that $H^{1}(L, E)_{0}=0$ for such $L-F$ modules. For cocycles of degree $\leqq-2$, we note that it was proved in $\S I I$ that there are no such cocycles in a onedimensional $L-F$ module over the contact algebra.

REMARK. Specific knowledge on the Spencer cohomology groups could have been used in parts (a) through (d) to give an alternate method of handling the cocycles of nonnegative degree.

IV. An application. We conclude with a simple application of cohomology to reducibility. Recall that when $L$ is a finite-dimensional simple Lie algebra and $M$ is a finite-dimensional module, $H^{1}(L, M)=0$ (Whitehead's lemma). It is an easy consequence of this that finite-dimensional modules over simple Lie algebras are completely reducible. In the infinite-dimensional case, the first cohomology group no longer vanishes and, in fact, we do not have complete reducibility.

THEOREM 6. Finite-dimensional $L-F$ modules are completely reducible.

Proof. Let $F \subset E$ be an $L-F$ submodule of the finite-dimensional $L-F$ module $E$. Let $G$ be the quotient $L-F$ module. $G$ is obtained by choosing a basis for a complement to $F / F^{0}$ in $E / E^{0}$ and taking the $L-F$ module generated by this basis. (It 
is clear that the quotient is a module over $F$ and that the imprimitivity property ((3) of Definition 3) is preserved on passing to a quotient.) The transitivity property which is needed follows from finite-dimensionality (Krull's lemma). We will use a modification of the proof given in Séminaire Sophus Lie (1955/1956).

Thus we have the exact sequence of $L-F$ modules,

$$
0 \rightarrow F \rightarrow E \rightarrow G \rightarrow 0 \text {. }
$$

This gives the exact sequence

$$
0 \rightarrow \operatorname{Hom}(G, F) \rightarrow \operatorname{Hom}(G, E) \rightarrow \operatorname{Hom}(G, G) \rightarrow 0
$$

where $\operatorname{Hom}(A, B)$ means all continuous linear maps (not necessarily module homomorphisms). This is obvious because the first sequence splits as vector spaces. To show there is a complement to $F$ in $E$ we must show that the sequence

$$
0 \rightarrow F \rightarrow E \rightarrow G \rightarrow 0
$$

splits as $L-F$ modules. That is, that there exists an $L-F$ module homomorphism from $G$ to $E$ which is an inverse to the projection of $E$ onto $G$. This in turn will follow as we can show that the sequence

$$
0 \rightarrow \operatorname{Hom}_{L-F}(G, F) \rightarrow \operatorname{Hom}_{L-F}(G, E) \rightarrow \operatorname{Hom}_{L-F}(G, G) \rightarrow 0
$$

is exact, where $\operatorname{Hom}_{L-F}(A, B)$ means all $L-F$ module homomorphisms. For then we can conclude that the identity map from $G$ to $G$, an $L-F$ module homomorphism, is the image of some $L-F$ module homomorphism from $G$ to $E$, as was desired.

To prove this, we will use cohomology. First of all, $\operatorname{Hom}(A, B)$ is an $L-F$ module: if $u \in \operatorname{Hom}(A, B)$,

$$
(\theta(x) u)(a)=\theta(x) u(a)-u(\theta(x) a)
$$

as usual; if $f \in F\left\{L / L^{0}\right\},(f u)(a)=f(u(a))$ where multiplication is as in $B$;

$$
\begin{aligned}
(\theta(x)(f u))(a) & =(x(f) u)(a)+f(\theta(x) u) a \\
& =x(f) u(a)+f \theta(x)(u(a))-f u(\theta(x) a) \\
& =\theta(x)(f u(a))-f u(\theta(x) a) \\
& =x(f) u(a)+f \theta(x) u(a)-f u \theta(x) a,
\end{aligned}
$$

which shows that this is an $L-F$ module. $\operatorname{Hom}(A, B)$ is not necessarily a free or finite-dimensional $L-F$ module. However, it is a transitive $L-F$ module, as is easily seen because $F^{0} \operatorname{Hom}(A, B)$ consists of homomorphisms that carry $A$ into $B^{0}$. Furthermore, it is generated by terms in a complement to $F^{0} \operatorname{Hom}(A, B)$, i.e., it satisfies the hypotheses of Theorem 2. (Cf. Remark following Theorem 2.)

Now consider the following long exact sequence of cohomology groups associated to $\left(^{*}\right)$ :

$$
\begin{aligned}
0 \rightarrow H^{0}(L, \operatorname{Hom}(G, F))_{0} \rightarrow H^{0}(L, \operatorname{Hom}(G, E))_{0} \\
\rightarrow H^{0}(L, \operatorname{Hom}(G, G))_{0} \rightarrow H^{1}(L, \operatorname{Hom}(G, F))_{0} \rightarrow \cdots,
\end{aligned}
$$


where the subscript zero means that we are considering only cocycles of degree at least zero (recall that the coboundary operator preserves the filtration degree). We have

$$
H^{1}(L, \operatorname{Hom}(G, F))_{0}=0
$$

because Hom $(G, F)$ is a transitive $L-F$ module (Theorem 2). This implies that

$$
H^{\circ}(L, \operatorname{Hom}(G, E))_{0} \rightarrow H^{0}(L, \operatorname{Hom}(G, G))_{0}
$$

is a surjection. But $H^{\circ}(L, M)_{0}$ is just the elements in $M$ of degree $\geqq$ zero that are invariant under $L$. The identity map from $G$ to itself is invariant under the action of $L$ and so is in $H^{\circ}(L, \operatorname{Hom}(G, G))_{0}$. Hence it must be the image of some $L-F$ module homomorphism from $G$ to $E$ under composition. Hence $F$ has a complement in E. Q.E.D.

\section{BIBLIOGRAPHY}

1. V. Guillemin and S. Sternberg, An algebraic model of transitive differential geometry, Bull. Amer. Math. Soc. 70 (1964), 16-47.

2. D. S. Rim, Deformations of filtered algebras. (I), Ann. of Math. 83 (1966), 339-357.

3. I. M. Singer and S. Sternberg, On the infinite groups of Lie and Cartan. (I), J. Analyse Math. 15 (1965), 1-114.

4. S. Kobayashi and T. Nagano, On filtered Lie algebras and geometric structures. IV, J. Math. Mech. 15 (1966), 163-176.

5. V. Guillemin, D. Quillen, and S. Sternberg, Classification of the complex primitive infinite pseudogroups, Proc. Nat. Acad. Sci. U. S. A. 55 (1966), 687-690.

6. G. Mackey, Infinite-dimensional group representations, Bull. Amer. Math. Soc. 69 (1963), 642-646.

7. V. Guillemin, D. C. Spencer, and S. Sternberg, Representation theory for transitive modules. I, (to appear).

8. A. Nijenhuis and R. W. Richardson, Jr., Cohomology and deformations in graded Lie algebras, Bull. Amer. Math. Soc. 72 (1966), 1-29.

9. S. Sternberg, Notes on transitive geometry, Harvard Univ., Cambridge, Mass., 1967.

10. C. Freifeld, The cohomology theory of transitive modules over the primitive infinite Lie algebras, Thesis, Harvard Univ., Cambridge, Mass., 1968.

11. - On transitive subalgebras of filtered Lie algebras, (in preparation).

HARVARD UNIVERSITY,

CAMbridge, MASSACHUSETtS 